\title{
Thermal transport properties of functionally graded carbon aerogels
}

\author{
Frank Hemberger ${ }^{*}$ \\ Sebastian Weis \\ Gudrun Reichenauer \\ Hans - Peter Ebert
}

Paper presented at $18^{\text {th }}$ European Conference on Thermopysical Properties, August 31 - September 4, 2008, Pau, France

Bavarian Center for Applied Energy Research (ZAE Bayern)

Functional Materials for Energy Technology

Am Hubland

97074 Würzburg

Germany

${ }^{*}$ : Corresponding author:

email: hemberger@zae.uni-wuerzburg.de

phone: 00499317056426

fax: 00499317056460 


\begin{abstract}
Novel graded carbon aerogels were synthesized to study the impact of different synthesis parameters on the material properties on a single sample and to test a new, locally resolved thermal conductivity measurement technique. Two identical cylindrical aerogels with a graded structure along the main cylindrical axis were synthesized. Along the gradient with an extension of about $20 \mathrm{~mm}$ the densities range from 240 to $370 \mathrm{~kg} \cdot \mathrm{m}^{-3}$ and the effective pore diameter determined via small angle X-ray scattering and SEM increase systematically from 70 up to $11000 \mathrm{~nm}$. One specimen was cut perpendicular to the cylinder axis into disc shaped samples; their thermal conductivities in argon atmosphere as determined via standard laser flash range from 0.06 to 0.12 $\mathrm{W} \cdot \mathrm{m}^{-1} \cdot \mathrm{K}^{-1}$ at $600^{\circ} \mathrm{C}$. The second specimen, cut to obtain a sample with the gradient in plane, was investigated with a spatially resolved laser flash technique at ambient conditions. The results of the two different techniques are compared and discussed in detail.
\end{abstract}

KEY WORDS: functionally graded, nanoporous materials, thermal diffusivity, thermal conductivity, local measurement 


\section{INTRODUCTION}

While functional graded materials (FGM) are not new, the concept having been used for millennia in steel production, graded structures are of increasing interest for both, technical application and academic research [1]. As a technical example, a functional graded thermal insulation can offer several advantages over a homogeneous material. The insulation can combine regions with good mechanical properties for mounting purposes and regions with a high porosity for better thermal performance. An ideal insulation material possesses a gradient in porosity and microstructure providing to a temperature gradient in the insulation with minimal heat transport.

Although the term FGM is frequently used for composite materials consisting of two or more different material phases, a FGM could also been synthesized from a single phase. An attractive approach from the research point of view is the realization of a wide variation of a specific material property in only one specimen. This approach allows on one hand the exclusion of many sources of uncertainty upon sample preparation in lab-scale and thus improves the comparability of the experimental data; on the other hand it can be used for a fast screening of material properties as function of a given synthesis parameter.

In this work the specific advantages of this approach is demonstrated on a functional graded carbon aerogel with a variation in pore size and density in one dimension. The graded carbon aerogels were synthesized by a novel modified sol-gel process. The influence of the gradient in morphology on the thermal transport properties of the graded carbon aerogel is investigated. The main focus of our investigations is the determination of the local thermal conductivity of the samples as a function of temperature and gas pressure; the later property is closely related to the local average pore diameter of the prepared graded carbon aerogels. The objective of this paper is to examine the option of a local determination of the thermal diffusivity of a graded specimen with a modified laser flash technique by comparing the data to those received with a conventional measurement setup and to results derived by a numerical simulation of the experiments. Main advantage of the proposed local determination of the thermal diffusivity would be a further simplification and acceleration of the specimen characterization upon the development of new materials.

\section{THEORETICAL BACKGROUND}

\subsection{Heat transport in porous media}

The effective total thermal conductivity $\lambda_{\text {eff }}$ of a porous material can be described in a good approximation as sum of four terms:

$$
\lambda_{\mathrm{eff}}=\lambda_{\mathrm{s}}+\lambda_{\mathrm{r}}+\lambda_{\mathrm{g}}+\lambda_{\mathrm{c}}
$$

were $\lambda_{\mathrm{s}}$ is the thermal conductivity related to the heat transport via the solid phase, $\lambda_{\mathrm{r}}$ is the radiative conductivity within porous optically thick materials and $\lambda_{\mathrm{g}}$ is the thermal conductivity of the gas within the pores. In addition, heat can be transferred via a local interaction between the solid and the gaseous phase leading to an additional coupling term, $\lambda_{\mathrm{c}}$. The later describes the increase of heat transfer in porous media consisting of particles, e.g. spheres or fibers, with a relatively high thermal conductivity compared to the surrounding phase (e.g. gas in 
pores) due to bypassing the thermal resistance of the point-shaped contacts between the particles by the pore gas or by thermal radiation.

\subsection{Contribution of the pore gas to the total effective heat transfer}

The focus in this work is on the contribution of the pore gas to the heat transport, since the thermal conductivity as function of gas pressure and the effective pore diameter of an open porous material are closely related. The corresponding thermal conductivity $\lambda_{\mathrm{g}}$ of the pore gas can be described according to Ref. 2 as

$$
\lambda_{\mathrm{g}}\left(T, p_{g}\right)=\frac{\Pi \cdot \lambda_{\mathrm{g}, 0}(T)}{1+\frac{\sqrt{2} \cdot \beta \cdot k_{\mathrm{B}}}{\sigma_{0}} \cdot \frac{T}{D \cdot p_{\mathrm{g}}}},
$$

were $\lambda_{\mathrm{g}, 0}$ is the thermal conductivity of the free gas, $\Pi$ is the porosity, $\sigma_{0}$ is the cross-section of the gas molecules, $k_{\mathrm{B}}$ is the Stefan-Boltzmann constant, $p_{\mathrm{g}}$ is the gas pressure and $D$ is the effective pore diameter of the porous media. The constant $\beta$ can be calculated by

$$
\beta=\frac{5 \cdot \pi}{32} \cdot \frac{2-\alpha}{\alpha} \cdot \frac{9 \cdot \gamma-5}{\gamma+1}
$$

with $\alpha$ the accommodation coefficient and $\gamma$ the adiabatic coefficient. The Knudsen number is defined as

$$
K n=l_{g} / D
$$

were the mean free path of the gas molecules can be calculated by

$$
l_{g}=\frac{k_{B} \cdot T}{\sqrt{2} \cdot \sigma_{0} \cdot p} .
$$

In Fig. 1 the gaseous thermal conductivity $\lambda_{\mathrm{g}}$ (according to Eq. (2)) is depicted as a function of the pore diameter $D$ for a temperature $T=300 \mathrm{~K}$ and constant gas pressure $p_{g}$. For large pore sizes $\lambda_{\mathrm{g}}$ becomes equal to $\Pi \cdot \lambda_{g, 0}$, i.e. the thermal conductivity of the free gas weighted with the porosity. In this limit the mean free path of the gas molecules is much smaller then the pore dimensions. For small pore sizes $D$ for which the mean free path of the molecules in pores is much smaller then the pore dimension, $\lambda_{\mathrm{g}}$ vanishes because probability for moleculemolecule interactions (i.e. gas phase transport) becomes negligible.

\section{GRADED CARBON AEROGEL - Preparation and structural properties}

\subsection{Sol-Gel Process}

A well established technique for the preparation of porous materials is the wet chemical sol-gel process. Starting from a chemical solution a sol is forming with the sol particles subsequently interconnecting to a three- 
dimensional network embedded in a liquid phase, the so called gel. Via a drying step the liquid phase is removed from the gel thus leaving a porous solid, the so called aerogel, behind [3].

The synthesis of homogeneous monolithic porous organic solids from resorcinol formaldehyde solutions is well established $[4,5]$. The size of the particles forming the backbone can be adjusted by the type and concentration of catalyst in the starting solution; the density of the resulting porous solid is controlled by the concentration of reactants in the starting solution and the shrinkage upon drying.

In this work a graded porous organic solid, using a starting solution of resorcinol (1,3-dihydroxybenzene) and formaldehyde mixed in a molar ratio of 1:2, was synthesized. The solution was diluted with de-ionized water to adjust the mass ratio of formaldehyde plus resorcinol to the total mass of the initial solution to $30 \%$. Sodium carbonate was added as a catalyst. The resorcinol-to-catalyst ratio (R/C-ratio) was set to 2000. After mixing, the solution was poured into an analytical glass vial $(1.7 \mathrm{~cm}$ in diameter). For the preparation of the gradient, diluted acid was layered on top of the solution. The acid was then allowed to diffuse into the underlying solution for several days at $20^{\circ} \mathrm{C}$. This affects the local concentration and composition of the catalyst and changes the mass ratio as the initial solution is locally further diluted. After the diffusion period the gelation of the sol is accelerated and completed by raising the storage temperature for several hours. After an exchange of the pore liquid water for ethanol the graded resorcinol formaldehyde (RF) aerogels were dried. The combination of a gradient in catalyst concentration and moderate dilution of the starting solution in direction of the diffusion front results in an organic aerogel with both, a backbone particle size and density gradient. Since at given density the average pore size is directly related to the average particle size a significant gradient in pore size is expected.

\subsection{Preparation of the graded carbon aerogel specimen investigated}

Two identical cylindrical graded RF-aerogel samples (gradient along the cylinder axis) were prepared. One of the samples was used to cut 15 disc-shaped specimens with a diamond precision saw. The thickness of these specimens was about $0.6 \mathrm{~mm}$. The direction of the gradient was oriented perpendicular to the surface of these samples. These samples will further be called "homogeneous specimen".

From the second sample, three specimens were cut parallel to the cylinder axis. Thus the gradient is oriented parallel to the sample surface. The thickness of these samples is also about $0.6 \mathrm{~mm}$. These specimens will further be called "graded specimen". In Fig. 2 the organic samples synthesized as well as the different types of specimen cut for the investigation of the thermal transport properties of the carbon derivatives are depicted. The graded structure in the organic RF-aerogel can easily seen by the color change of the specimen.

After the preparation all specimen were pyrolysed at $800^{\circ} \mathrm{C}$ under argon atmosphere to yield carbon aerogels; i.e. upon this process step the organic solid backbone is totally converted into a pure carbon structure with almost the same morphology. Upon pyrolysis a linear shrinkage of the sample of about $20 \%$ occurs. The thickness of the specimens is consequently reduced to about $0.5 \mathrm{~mm}$. In Fig. 3 one of the graded carbon specimen is depicted. The graded structural regime is about $20 \mathrm{~mm}$ wide. The region in the lower part of the specimen is homogeneous with respect to both, its density and microstructure. 


\section{EXPERIMENTAL AND NUMERICAL TECHNIQUES}

\subsection{Determination of the thermal conductivity}

The thermal conductivity was determined indirectly by using the laser flash method [6]. During the laser flash experiment one side of the specimen investigated is heated by a short laser pulse. The heat diffuses through the specimen and causes a rise of the temperature on the opposite side of the specimen. This temperature rise is monitored as a function of time with an infrared detector and used for the evaluation of the thermal diffusivity. A detailed description of the evaluation procedure to obtain the thermal diffusivity of the investigated specimen from the temperature rise is given in Ref. 7. Finally, the thermal diffusivity $a$ is converted into a total effective thermal conductivity $\lambda$ using:

$$
\lambda\left(T, p_{g}\right)=a\left(T, p_{g}\right) \cdot \rho(T) \cdot c_{p}(T)
$$

with $p_{\mathrm{g}}$ the gas pressure in the pores of the porous material investigated. To calculate the thermal conductivity the material density $\rho$ and the specific heat capacity $c_{\mathrm{p}}$ have to be known or determined separately. In this work the density was determined by the weight of the specimen and its dimensions, the thermal expansion of the carbon aerogel was neglected in this work because it was measured to be below $0.1 \%$ at $600^{\circ} \mathrm{C}$ [8]; for $c_{\mathrm{p}}$ of the carbon aerogel specimens, literature values for graphite were used [9].

The experimental setup used in this work for the determination of the thermal diffusivity via the standard laser flash method is also described in Ref. 7.

For the local determination of the thermal diffusivity of the graded specimen a technique described in Ref. 10 was used in this work. The key feature is the position control of the specimen in the plane perpendicular to the laser beam and the IR-detection unit. The mechanical precision of the position control is better than $100 \mu \mathrm{m}$. The IR-detection unit consists of a MCT-detector, a polycrystalline IR-fiber with a diameter of $1 \mathrm{~mm}$, and a system to focus on the sample surface. The effective numerical aperture of 0.25 for the fiber and a maximum distance of $1 \mathrm{~mm}$ between the fiber and specimen lead to a detection area with a diameter of $1.5 \mathrm{~mm}$ in maximum. For the evaluation procedure, the specimen is assumed to be homogeneous and isotropic across the detection area and Eq. 2 and Eq. 6 can be applied. The minimum uncertainty for the determination of the thermal diffusivity via this experimental setup is $5 \%$ [10].

\subsection{Numerical simulation of the laser flash experiment}

Time dependent numerical simulations of the experiment in two dimensions were performed to test the reliability of the locally determinate thermal diffusivity data of the graded specimen. For this task the commercial computer program FlexPDE 5.0, based on the finite element method, was used (www.pdesolutions.com). An overview over the geometry of the model implemented in the simulation routine is depicted in Fig. 4. For the correct consideration of heat losses occurring in the experiment, the surroundings of the specimen was also included in the numerical model; the specimen is placed in an environment with the thermal conductivity and the heat capacity of air. To consider the heat losses by radiation from the specimen surface occurring during the experiment, there is a reasonable temperature flux on the specimen surface implemented into the simulation. This flux is proportional to the temperature of the specimen surface in respect to the starting temperature. 
Like the real experiment, the simulation starts with a temperature pulse on the front side of the specimen of 0.3 ms duration. For the result of the simulation the time dependant temperature rise on the backside of the sample is calculated by integration over the temperature field and a subsequent normalization. This temperature rise is then evaluated by a standard analytical model $[6,7]$ for the laser flash experiment to derive the thermal diffusivity $a$. The precision of the numerical simulation was tested for a standard laser flash experiment on a homogeneous specimen. The resulting difference between the value for $a$ used in the simulation and the corresponding values derived via the evaluation of the simulated temperature rise was found to be below $2 \%$.

As input data for the simulation of the local determination of the thermal diffusivity, the experimentally determined values for the thermal conductivity and the measured values for the density of the homogeneous specimen were used. The values for both properties were given as function of the position in z-direction for the correct simulation of the graded structure.

In Fig. 5 a typical result of the numerical simulation for the temperature rise on the backside of the specimen as function of time is shown. Also shown is the result of the evaluation of this simulated laser flash experiment by the used standard analytical model $[6,7]$.

\subsection{Morphological characterization of the samples}

The morphology of the gradient was investigated by a combination of scanning electron microscopy (SEM) and of small angle X-ray scattering (SAXS); the scattering measurements were performed with synchrotron radiation at the instrument JUSIFA (Hasylab, Hambug, Germany).

The effective pore diameter $D$ for regions with backbone particle diameters below $2000 \mathrm{~nm}$ in the graded carbon aerogel can be calculated by

$$
D=\frac{4 \cdot V_{P}}{S_{e x t}}=\frac{4}{S_{e x t}} \cdot\left(\frac{1}{\rho}-\frac{1}{\rho_{s}}\right),
$$

with the external specific surface area $S_{\text {ext }}$ determined by SAXS data; $\rho$ is the bulk density of the specimen and $\rho_{\mathrm{s}}$ is the density of the solid backbone; $V_{\mathrm{P}}$ is the pore volume.

The effective pore diameter for regions with particle diameters above $2000 \mathrm{~nm}$ was determined via imaging analysis by SEM [11].

\section{RESULTS AND DISCUSSION}

\subsection{Morphology of the gradient}

In Fig. 6 the size of the particles forming the carbon backbone is plotted as function of their position along the gradient. Also depicted in Fig. 6 is the effective pore diameter $D$ calculated from the external specific surface using Eq. 7 and imaging analysis of the SEM pictures.

The plot reveals a strong monotonic increase in pore size from 70 to $11000 \mathrm{~nm}$ along the gradient with a width of $20 \mathrm{~mm}$ only. In the homogeneous regime (Fig. 6, left) the pore size is - within the combined uncertainty of the pore size determination and the reproducibility of the specimens of less than $\pm 10 \%$ - equal to the value determined for a non-graded specimen derived from the corresponding unmodified starting solution. At the 
opposite end of the gradient (Fig. 6, right) huge particle and pore sizes are present as expected for acid catalyzed carbon aerogels synthesized with a mass ratio below $40 \%$ [12]. The transition regime reflects the sensitivity of the gel formation on the continuous change in acid concentration.

In Fig. 7 the measured densities of the 15 homogeneous specimens are depicted as a function of their initial position in the specimen. The experimental density values were interpolated by a $4^{\text {th }}$ order polynomial in the range between position $0 \mathrm{~mm}$ and $20 \mathrm{~mm}$. The relative uncertainty of the density values is about $3 \%$. This curve is used for the calculation of the thermal conductivity according to Eq. 6 and for the numerical simulation of the laser flash experiment.

\subsection{Thermal conductivity of the specimen along the gradient}

The thermal conductivity of the 15 homogeneous specimens was determined at $300^{\circ} \mathrm{C}$ and $600^{\circ} \mathrm{C}$ both, in 0.1 MPa argon atmospheres as well as in the evacuated state (gas pressure below $1 \mathrm{~Pa}$ ). In Fig. 8 the results are depicted as a function of the position along the gradient. The relative uncertainty of the thermal conductivity values is about $10 \%$. A strong variation of the thermal conductivity with the position along the gradient is observed for the evacuated as well as the gas filled specimens.

The difference between the thermal conductivity values of the gas filled and the evacuated specimens is related to the contribution of the pore gas and potential coupling effects to the total effective thermal conductivity of the porous material. Using the relation between the effective pore diameter and its position along the gradient (Fig. 6), the gaseous conductivity as a function of the position along the gradient can be converted into a representation of the gaseous thermal conductivity as a function of pore diameter (Fig. 9). Also depicted in Fig. 9 is the theoretical expected contribution of the pore gas to the effective thermal conductivity, calculated according to Eq. 2 and 3. Hereby a cross section of $\sigma_{0}=3.6 \cdot 10^{-19} \mathrm{~m}^{2}$ for argon has been used. For the adiabatic coefficient $\gamma$ was set to $5 / 3$ and for the accommodation coefficient $\alpha=1$ was applied. For both, the experimental and the theoretical gaseous thermal conductivity a large increase of the gaseous thermal conductivity the with the pore size is found; this is due to the transition from heat transfer via molecular transport in pores with diameters much smaller than the mean free path of argon (i.e. $125 \mathrm{~nm}$ at $300^{\circ} \mathrm{C}$ and $190 \mathrm{~nm}$ at $600^{\circ} \mathrm{C}$ ) to diffusive heat transfer in pores much larger than the mean free path of the pore gas. The higher thermal conductivities derived for $600^{\circ} \mathrm{C}$ compared to the data determined for $300^{\circ} \mathrm{C}$ can be attributed to the slightly different heat capacities of the sample at 300 and $600{ }^{\circ} \mathrm{C}$. Striking is the fact that the experimentally deduced values are in all cases significantly higher that the ones expected theoretically for a pure gas phase transport. It therefore has to be assumed that strong coupling effects are present that result in local microscopic short circuits of the heat transfer and thus enhance the overall effective thermal conductivities. The steep increase at pore diameters above 10000 $\mathrm{nm}$ can not be explained by the theoretical model used in this work. A possible explanation would be a change of the inner morphology of the backbone particles without changes in the particle size and the bulk density of the investigated specimen.

\subsection{Spatially resolved thermal diffusivity of the graded specimen}

The thermal diffusivity of the homogeneous specimen and the local thermal diffusivity of the graded specimen were determined at ambient conditions. The results of the measurement are plotted as function of the average pore diameter in figure 10. The dataset show a good agreement for pore diameters larger than $200 \mathrm{~nm}$. This 
corresponds also to the region with the strongest morphology change along the gradient (see Fig. 6). The regime with pore diameters of about $10000 \mathrm{~nm}$ is the region with the lowest densities along the gradient. At this position the graded specimen investigated tends to be brittle. This indicates that the interconnectivity of the backbone particles in the material is here less pronounced and consequently lower values for the thermal diffusivity are observed in comparison to the homogeneous specimen.

For pore diameters below $200 \mathrm{~nm}$ significant differences between the two measurement series can be observed. In principle this difference could be caused by the method applied for the determination of the local thermal diffusivity. To check this hypothesis a numerical simulation of the modified laser flash experiment was performed to elucidate the impact of the experimental technique on the data extracted. The results of this simulation are plotted in Fig. 10. The graph shows that the simulated local determination of the thermal diffusivity yields the same results as derived with the standard laser flash experiment. Consequently, the modified laser flash experiment applied can be assumed to yield reliable values for the local thermal diffusivity.

A characteristic property of the specimen in the pore size range smaller $200 \mathrm{~nm}$ is its significantly higher shrinkage upon drying and thus a strong increase in density for pore sizes below $200 \mathrm{~nm}$; this is due to the capillary forces exerted by the pore liquid upon drying, that scale inversely with the pore diameter. Comparing the shrinkage and the thermal diffusivity as function of pore diameter seems to suggest a correlation between the change in shrinkage (and thus density) and the discrepancies in the results of the thermal diffusivities for pore diameters smaller than $200 \mathrm{~nm}$ (Fig. 11). In Fig. 12 a profile of the cross section of a cylindrical RF aerogel after drying process is depicted. The picture shows that the shrinkage in diameter (r-direction) is far stronger for the region with small pores below $200 \mathrm{~nm}$ (here: bottom) than for the region with the larger pores (here: upper dark and light part). If the non uniform shrinkage induces shrinkage in z-direction, the spatially resolved thermal diffusivity measurements performed perpendicular to the cylinder axis will sense a locally anisotropic thermal diffusivity. Considering the different orientations of the "homogenous" and "graded" with respect to the original cylindrically shaped, graded RF aerogel, it can be seen that the heat flow direction during the laser flash experiment on the homogeneous specimen is along the z-axis, while the laser flash experiment on the graded sample is probing the morphology perpendicular to the z-axis. Consequently, the differences in the measurement results is likely to reflect the additional anisotropy of the cylindrical shaped graded RF aerogel in the region with pores smaller than $200 \mathrm{~nm}$. It should be emphasized that this region is not identical to the region with the gradient along the z-axis in morphology.

\section{CONCLUSIONS}

The synthesis and characterization of functionally graded structures provides many advantages for both, academic research and material development. It was shown that it is possible to significantly reduce the efforts with respect to material synthesis upon screening of synthesis parameters by preparing only one graded sample with specific modified material properties.

The morphological characteristics can easily be determined via spatially resolved SEM or SAXS. Within the framework of this study it was shown that also the thermal transport properties can be determined fast and locally resolved within only one graded specimen. The measurements on only one carbon aerogel with a $20 \mathrm{~mm}$ wide gradient in pore size (ranging from 70 to $11000 \mathrm{~nm}$ ) and about a factor of 1.5 in density reveal a systematic 
increase in gaseous thermal conductivity with pore size as expected as well as an additional contribution to the heat transport via coupling that is on the same order of magnitude as the pure gas phase transport.

So far the results of the spatially resolved thermal transport measurements do not in all cases coincide with the classical approach; this can be due to anisotropies perpendicular to the direction of the gradient. This hypothesis will be investigated in the near future. 


\section{REFERENCES}

- [1] S. Suresh and A. Mortensen, Fundamentals of Functionally Graded Materials, Book 698 (IOM Communications Ltd, London, 1998), p. 5

- [2] M. G. Kaganer, Thermal Insulations in Cryogenic Engineering, (Israel Program for Scientific Translations, 1969)

- [3] G. Reichenauer, Aerogels, in Kirk-Othmer Encyclopedia of Chemical Technology (John Wiley \& Sons, Inc., 2008)

- $\quad$ [4] R. W. Pekala and F.M. Kong, J. De Physique 50:C433 (1989)

- [5] M. Wiener, G. Reichenauer, T. Sherb and J. Fricke, J. Non-Cryst. Solids, 350:126 (2004)

- [6] W. J. Parker et al., J. Appl. Phys. 32:1679 (1961)

- [7] O. Nilsson, H. Mehling, R. Horn, J. Fricke, R. Hofmann, S. G. Müller, R. Eckstein and D. Hofmann, High Temp. - High Press. 29:73 (1997)

- $\quad$ [8] J. Gross and J. Fricke, J. Non-Cryst. Solids, 186:301 (1995)

- [9] J. G. Hust, Standard Reference Materials: A Fine-Grained, Isotropic Graphite for Use as NBS Thermo-physical Property RM's from 5 to 2500 K, U.S. Department of Commerce, National Bureau of Standards, Special Publication 260-89 (1984)

- $\quad[10]$ F. Hemberger, H.-P. Ebert and J. Fricke, Int. J. Thermophys., 28:1509 (2007)

- [11] G. Reichenauer, U. Heinemann and H.-P. Ebert, Colloids and Surfaces A: Physicochem. Eng. Aspects 300 (2007) pp. 204-210

- $\quad$ [12] R. Brandt, R. Petricevic, H. Probstle and J. Fricke, J. Porous Materials 10:171 (2003) 


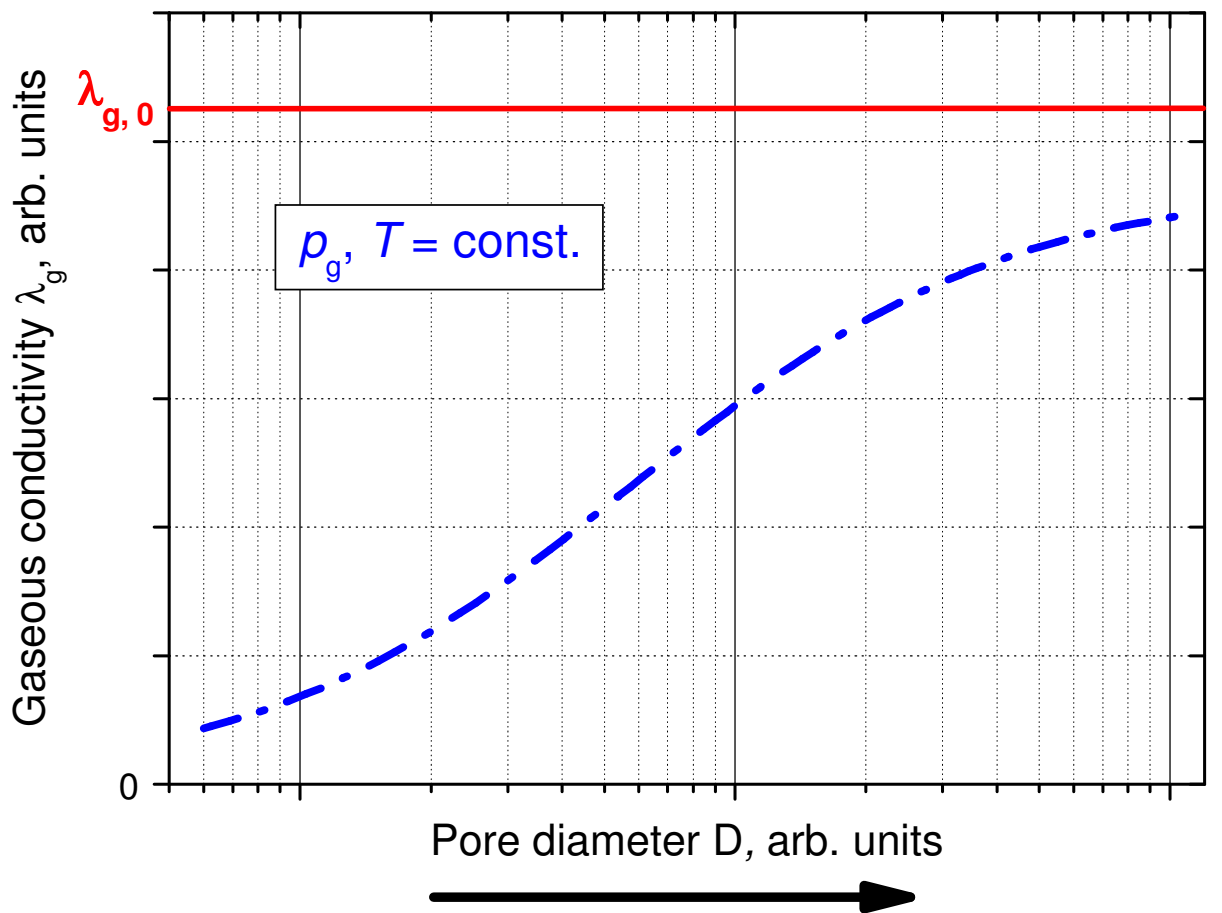

Figure 1: Thermal conductivity via the gas phase in the pores as a function of pore diameter $D$ for constant temperature and gas pressure. 
(2)

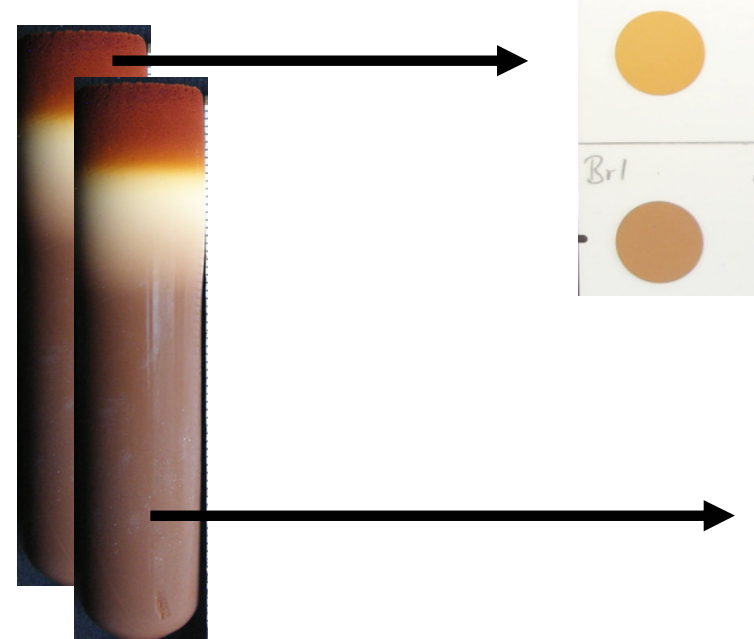

(1)

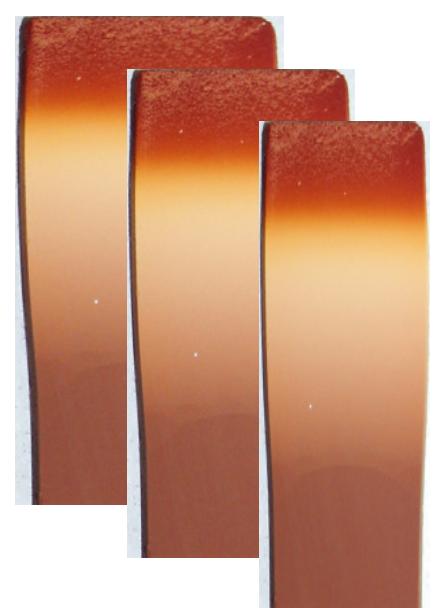

(3)

Figure 2: Cylindrical shaped RF aerogels synthesized (1). (2): 15 disc shaped, homogeneous specimens cut from one of the as prepared cylinders. (3): Three graded specimens cut from the second as prepared cylinder. The structural gradient becomes macroscopically visible through the color change. 


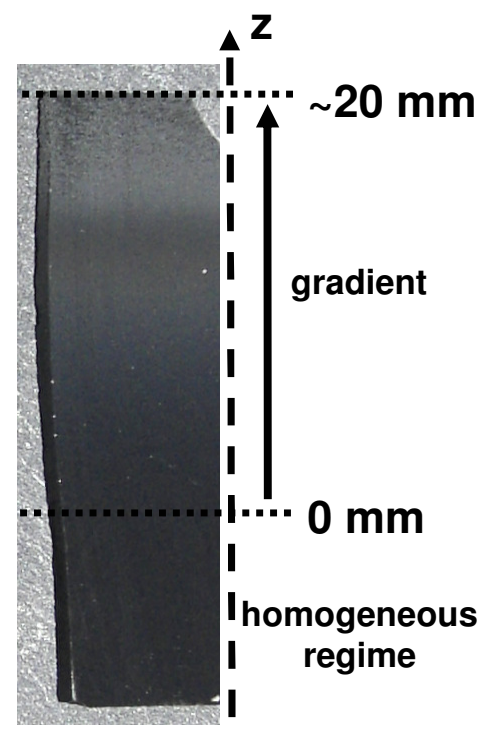

Figure 3: Graded carbon aerogel specimen after pyrolysis at $800^{\circ} \mathrm{C}$. The graded structure is about $20 \mathrm{~mm}$ in extension. 


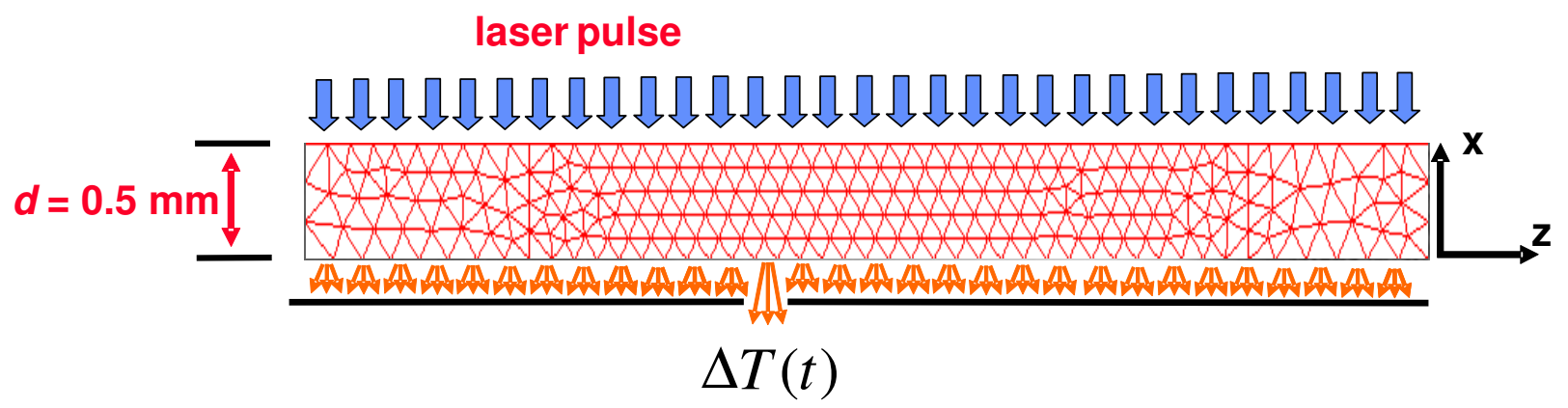

Figure 4: 2D model for the time dependent numerical simulation of the local determination of the thermal diffusivity $a$ of the graded specimen. The structural gradient is orientated along the $\mathrm{z}$-direction. The front side of the specimen (here: top) is fully irradiated by the laser pulse as in a standard laser flash experiment. The temperature as a function of time is monitored on the backside (here: bottom) at 25 locations. The surroundings of the specimen are not shown however they are included in the numerical model with the thermal conductivity and heat capacity of air. 


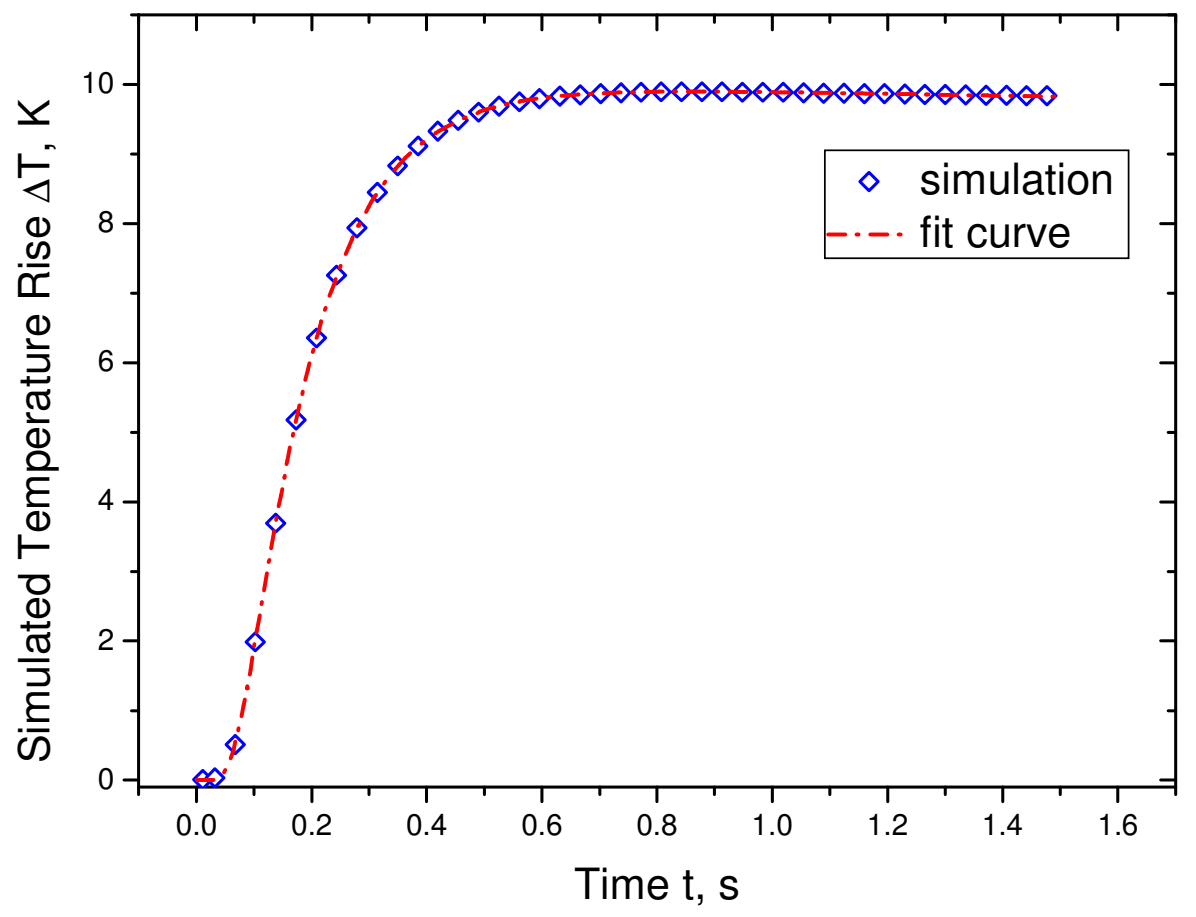

Figure 5: Typical result of the numerical simulation for the temperature rise on the backside of the investigated specimen during a laser flash experiment. Also depicted is the result of the fit curve according to the analytical model described in Ref. 6 and Ref. 7. 


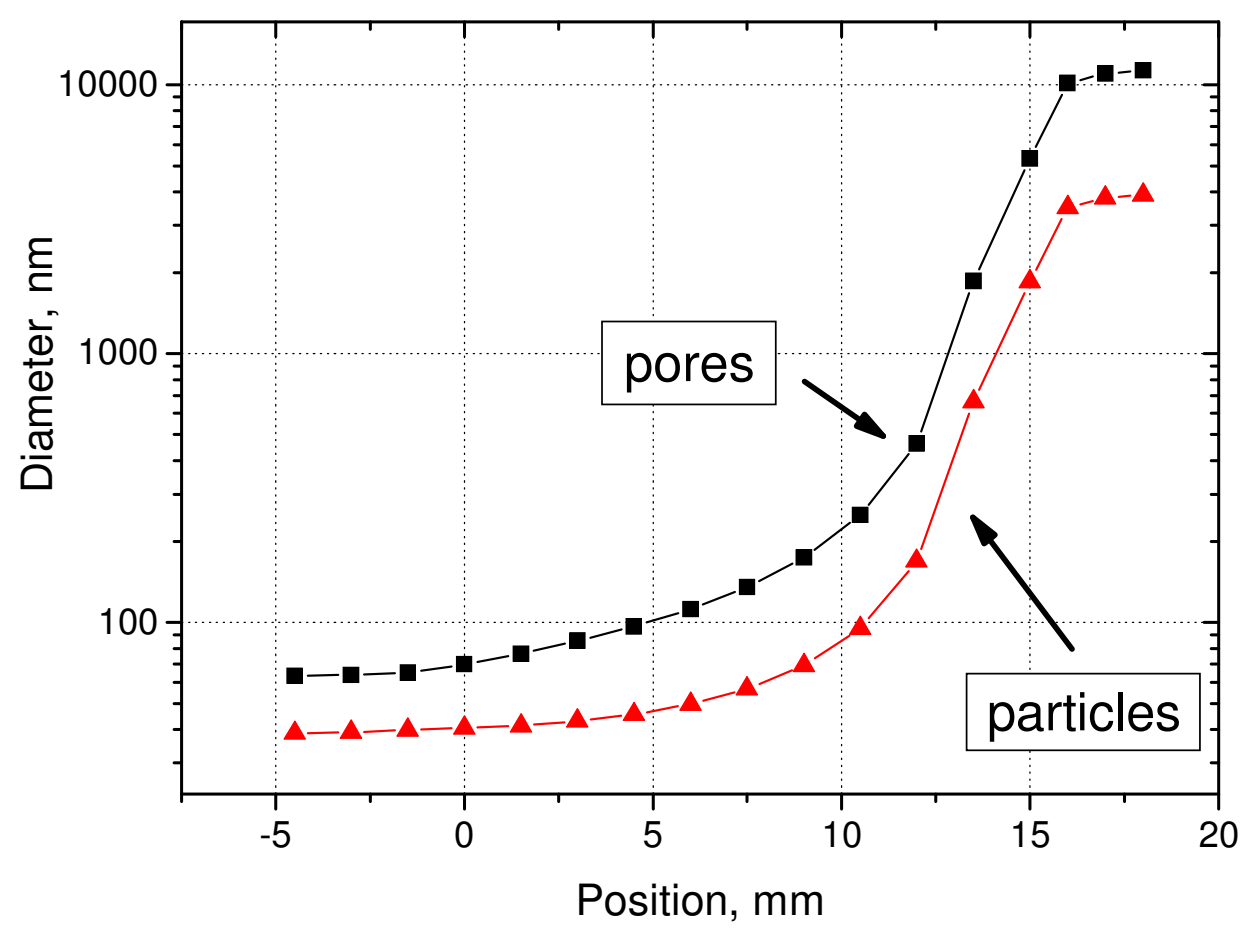

Figure 6: Particle size of the solid backbone and effective pore diameter of the graded carbon aerogel as function of the position along the gradient. 


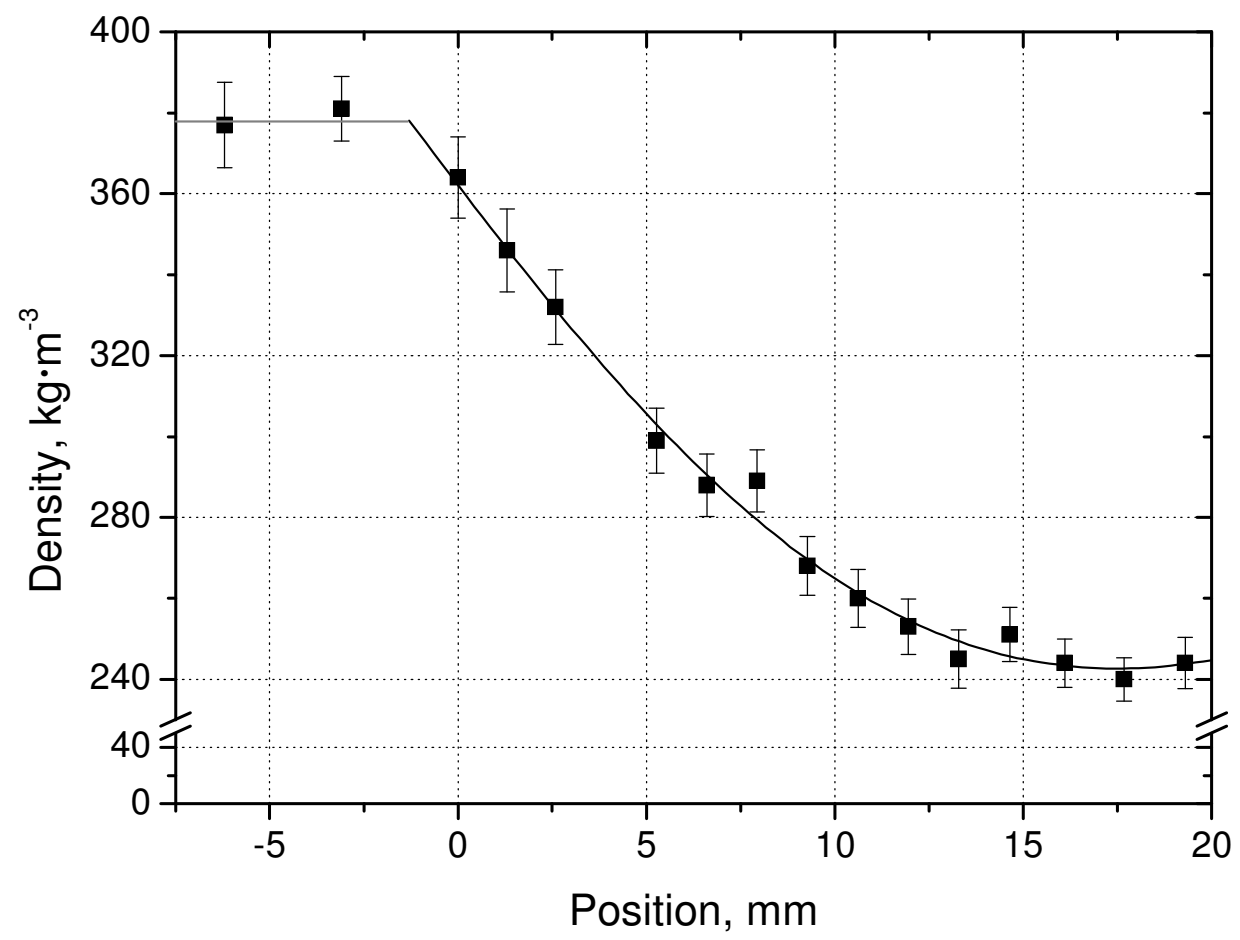

Figure 7: Density (symbols) of the homogeneous carbon specimens investigated as function of the position along the gradient. Also shown is the result of a non-linear fit with a polynomial of 4 th order (solid line). 


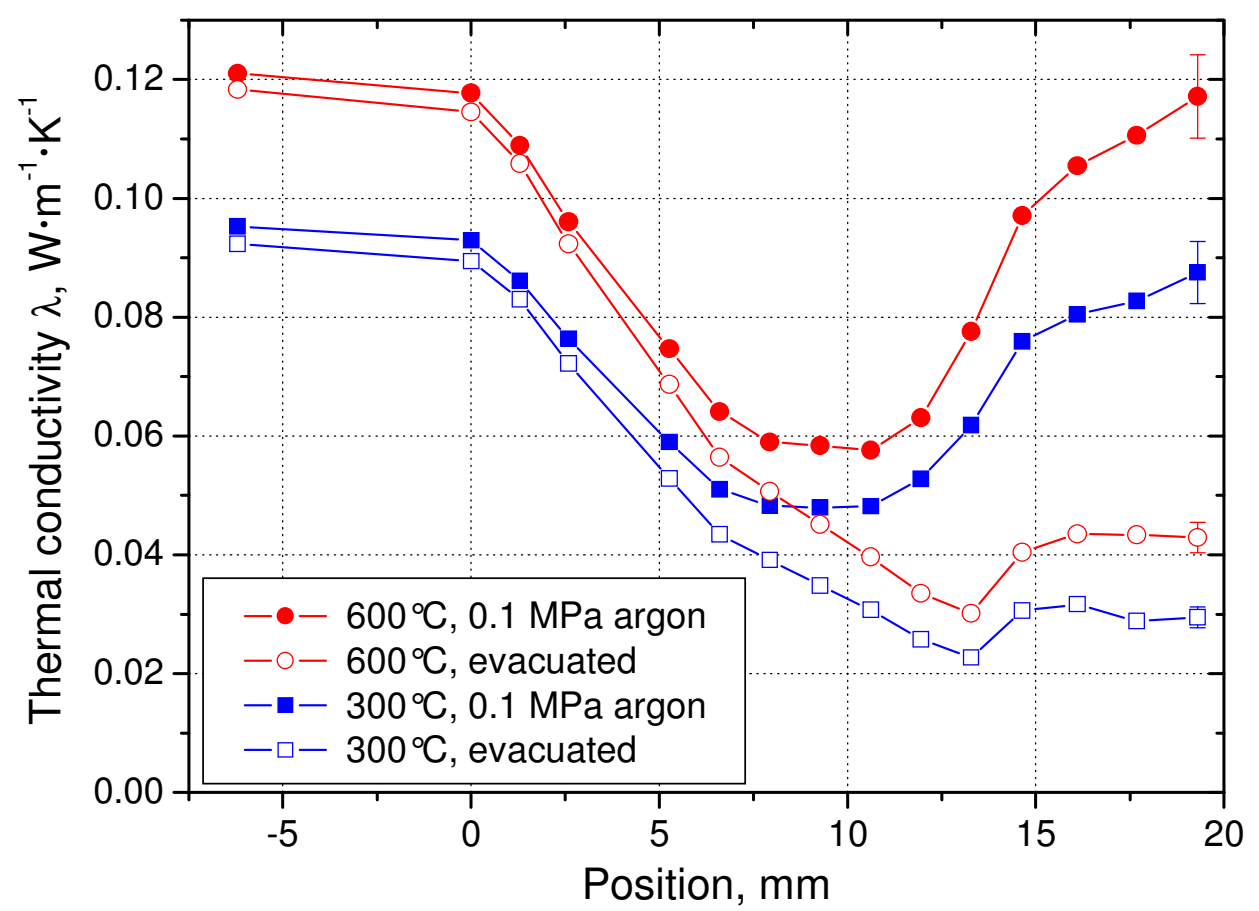

Figure 8: Thermal conductivity at $300^{\circ} \mathrm{C}$ and $600^{\circ} \mathrm{C}$ measured on the homogeneous carbon aerogel specimens as a function of their initial position in the graded carbon aerogel. Each specimen was measured under vacuum and an argon gas pressure of $0.1 \mathrm{MPa}$. The depicted error bars indicate the typical uncertainty of the values of $6 \%$. 


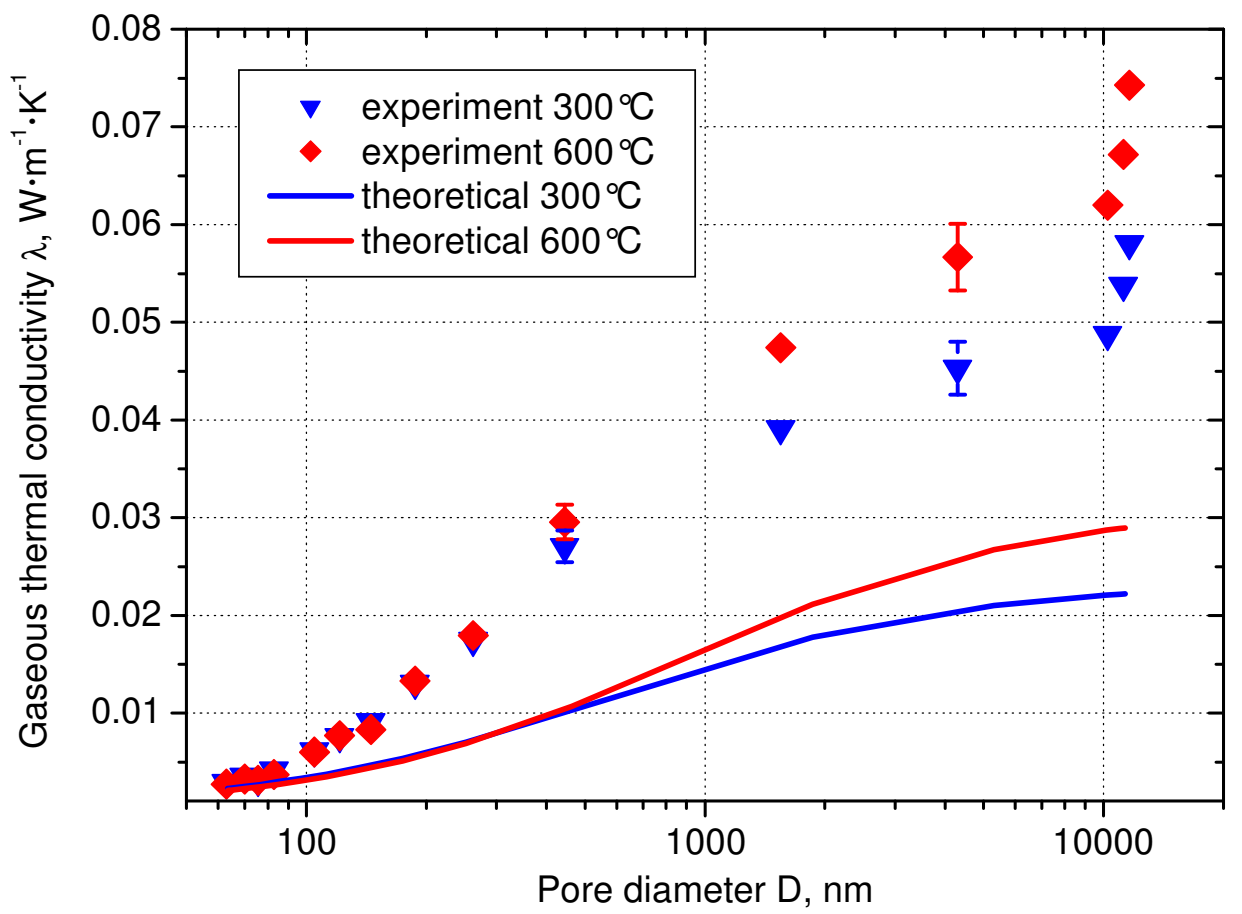

Figure 9: Difference between the thermal conductivity values measured at $0.1 \mathrm{MPa}$ in argon and in the evacuated state at $300^{\circ} \mathrm{C}$ and $600^{\circ} \mathrm{C}$ as a function of the pore diameter (see Fig. 5 and 7). Also depicted are the theoretically expected values (solid lines) calculated via Eq. (2). The error bars depicted represent the typical uncertainties of the experimental data. 


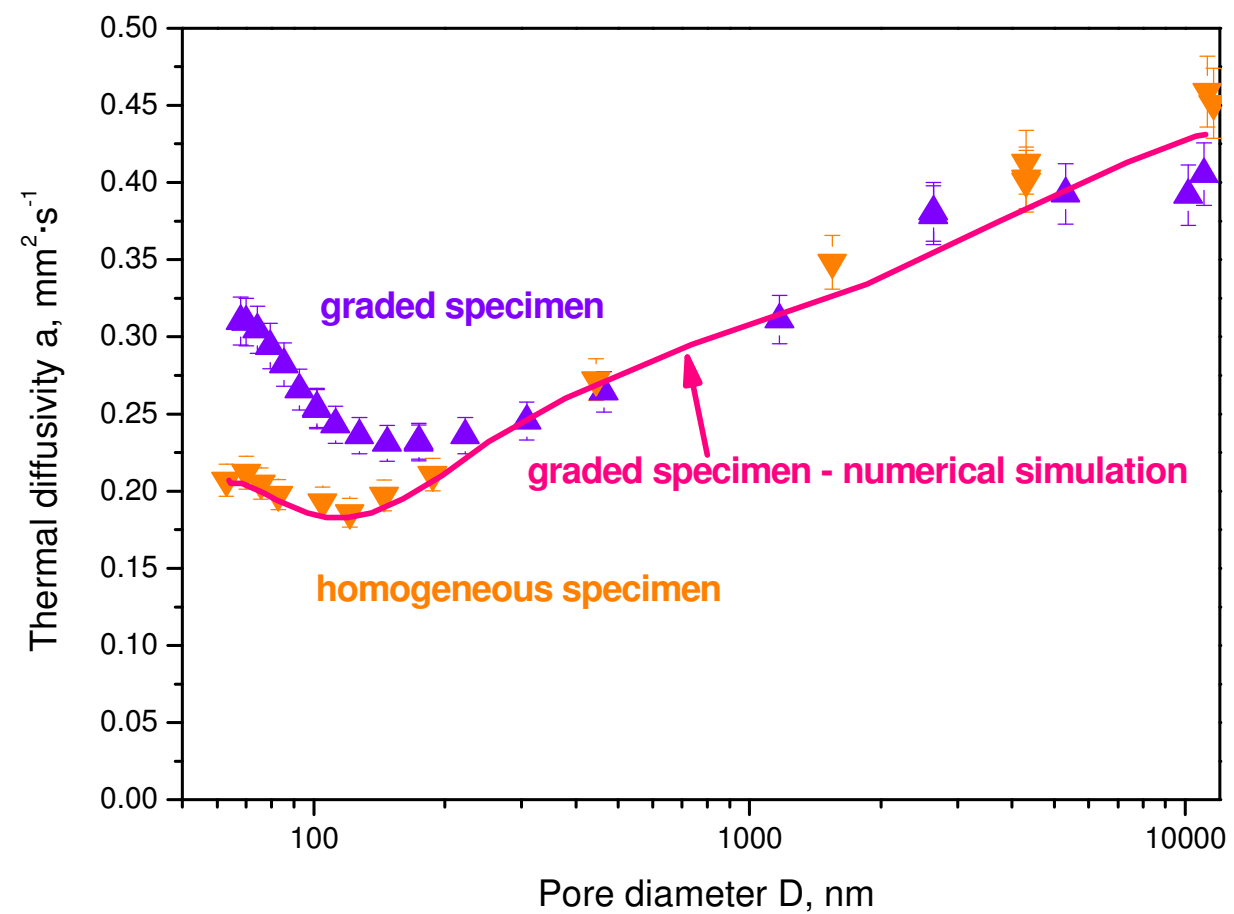

Figure 10: Comparison of the thermal diffusivities a measured for the homogeneous specimen and the corresponding graded specimen as a function of pore diameter. Also depicted are the results from a 2D numerical simulation of the local determination of $a$ for the graded specimen; hereby the values for $a$ measured on the homogeneous specimen were used as input data. 


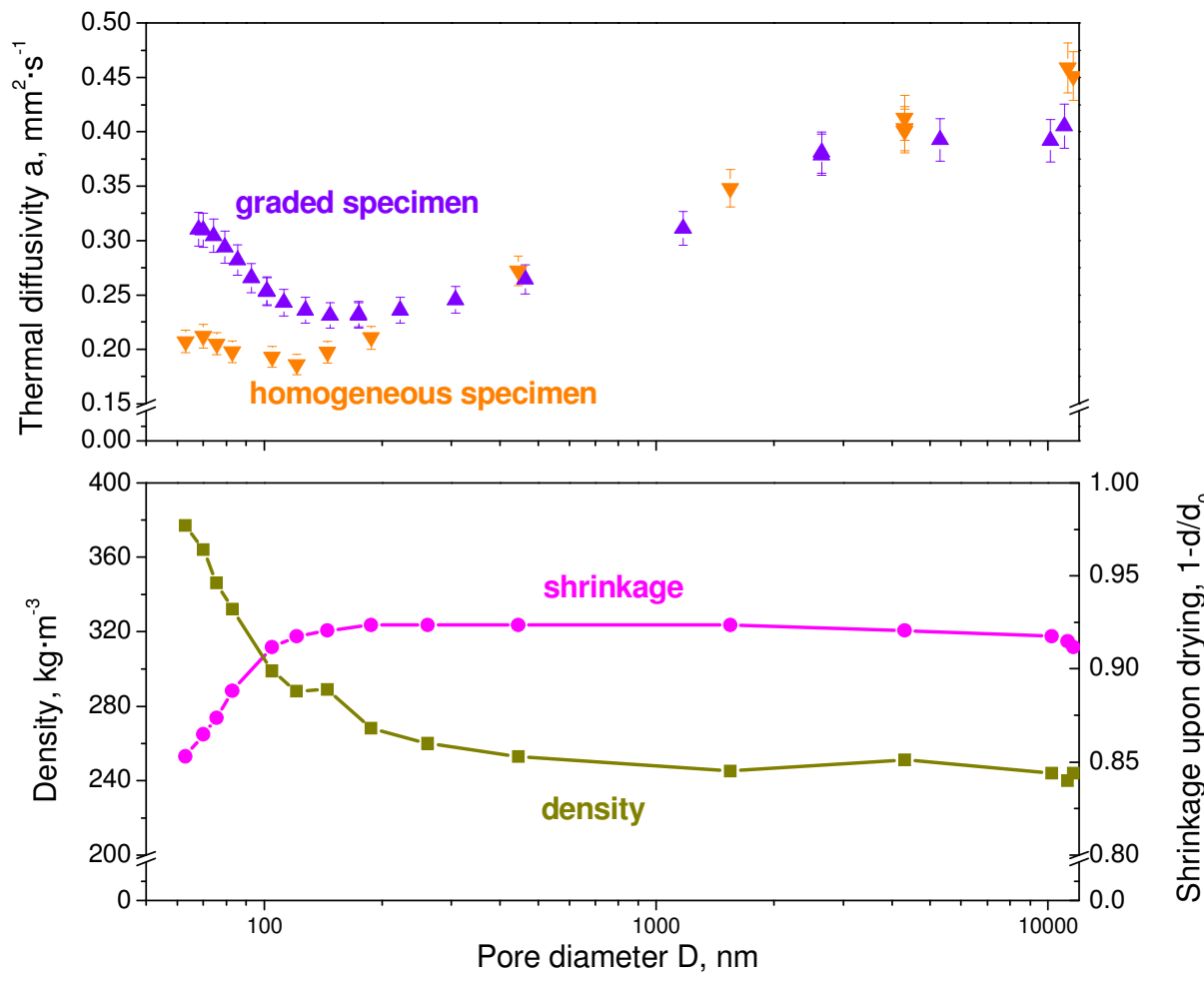

Figure 11: Correlation between discrepancies in thermal diffusivity (top) and shrinkage and thus change in material density upon drying as a function of pore diameter. 


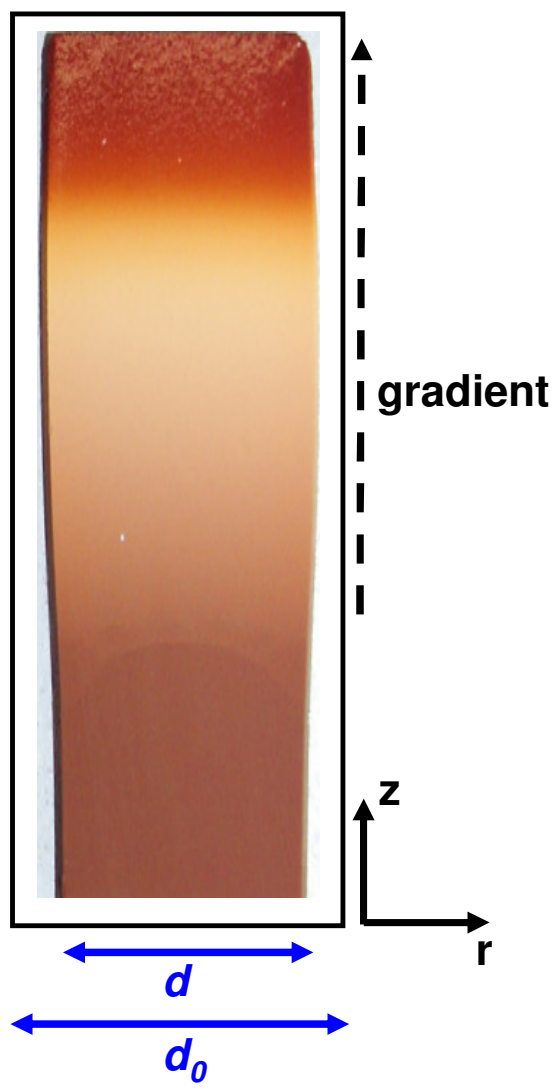

Figure 12: Non uniform shrinkage of the graded RF aerogel upon the drying process. The shrinkage is stronger in the lower region with the smaller pores than in the upper range that is characterized by larger pores. 